\title{
A Routing Strategy for Multihomed ISP to Mitigate the Impact of Overlay Traffic
}

\author{
Xun SHAO $^{\dagger a)}$, Student Member, Go HASEGAWA ${ }^{\dagger \dagger b)}$, Yoshiaki TANIGUCHI ${ }^{\dagger \dagger c)}$, \\ and Hirotaka NAKANO ${ }^{\dagger \dagger \mathrm{d})}$, Members
}

\begin{abstract}
SUMMARY Multihoming is widely used by Internet service providers (ISPs) to obtain improved performance and reliability when connecting to the Internet. Recently, the use of overlay routing for network application traffic is rapidly increasing. As a source of both routing oscillation and cost increases, overlay routing is known to bring challenges to ISPs. In this paper, we study the interaction between overlay routing and a multihomed ISP's routing strategy with a Nash game model, and propose a routing strategy for the multihomed ISP to alleviate the negative impact of overlay traffic. We prove that with the proposed routing strategy, the network routing game can always converge to a stable state, and the ISP can reduce costs to a relatively low level. From numerical simulations, we show the efficiency and convergence resulting from the proposed routing strategy. We also discuss the conditions under which the multihomed ISP can realize minimum cost by the proposed strategy.

key words: ISP, multihoming, overlay routing, routing game, Nash equilibrium
\end{abstract}

\section{Introduction}

Singlehoming is the most basic form of Internet connection, where an ISP uses a single transit provider to reach all destinations. However, singlehoming may lead to poor reliability and performance of the connection. To achieve improved reliability and performance, many Internet service providers (ISPs) now use multihoming. By employing advanced technologies, known as intelligent route control [1] and smart routing [2], a multihomed ISP can split traffic flow among multiple transit links based on cost and performance considerations.

Overlay routing, which can improve end-to-end performance in a network by changing its traffic routing at the application layer, is also rapidly increasing. This routing is shown to effectively solve some of the shortcomings of today's Internet. Measurements from [3] indicate that in the current Internet, a large percentage of flows can find a better route by relaying packets with the assistance of overlay nodes. Various overlay applications exist in today's Internet,

Manuscript received June 5, 2012.

Manuscript revised August 29, 2012.

${ }^{\dagger}$ The author is with the Graduate School of Information Science and Technology, Osaka University, Toyonaka-shi, 560-0043 Japan.

${ }^{\dagger}$ The author is with the Cybermedia Center, Osaka University, Toyonaka-shi, 560-0043 Japan.

a) E-mail: x-shao@ist.osaka-u.ac.jp

b) E-mail: hasegawa@cmc.osaka-u.ac.jp

c)E-mail: y-tanigu@cmc.osaka-u.ac.jp

d)E-mail: nakano@cmc.osaka-u.ac.jp

DOI: 10.1587/transinf.E96.D.193 such as P2P file sharing applications, service overlay networks [3] and CDN [4]. Although different kinds of overlay applications have different users and mechanisms, all their objectives are to optimize end-to-end performance for users of their own, so that their traffics behave selfishly.

[5] pointed out that a fundamental mismatch is found between the objectives of overlay routing and those of an ISP's traffic engineering. An overlay application looks to optimize the routes of its users, whereas an ISP's traffic engineering attempts to improve the entire network performance and reduce operational cost by considering all users (overlay and non-overlay). Since both processes optimize their associated routes over time, the interaction between their decisions can be seen as an iterative process. For example, in a certain step, overlay routing allocates traffic on links according to the end-to-end network performance perceived by its users, and in the next step the ISP reallocates the traffic to optimize its objectives. The interaction of such processes can be modeled by game-theoretic approaches [6]. Although the objective mismatch between overlay routing and ISP traffic engineering has been pointed out in previous studies, the majority of the research focuses on ISPs' intra-domain networks. Little work has been conducted on inter-domain traffic engineering such as multihoming.

In a typical multihoming network, a multihomed ISP connects to the Internet via multiple transit links provided by different transit providers. The transit providers may provide transit links with different capacities and prices. Here, we conceptualize the network as a model with parallel links, as shown in Fig. 1. The network contains non-overlay and overlay traffic. Non-overlay traffic routes are determined by the ISP only, whereas overlay traffic routes can also be determined by overlay applications. The ISP's routing decisions are always made such that its total cost is reduced, and the overlay applications always attempt to send traffic through links with minimum latency.

Specifically, in this work, we assume that the ISP's total cost is composed of two parts. When sending and receiving traffic through transit links, the ISP must pay the transit providers. This cost is referred to as monetary cost. In addition, the ISP must consider the average latency on each link when making routing decisions. The cost caused by link latency is referred to as latency cost, and is a measure of performance decrease. The total cost to the ISP is then defined as the weighted summation of monetary and latency costs. 


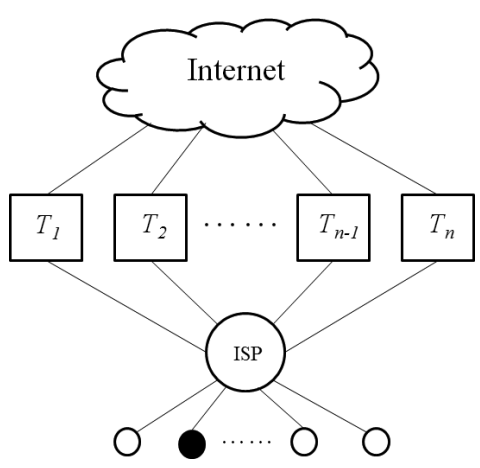

Fig. 1 Network model: $T_{i}, i=1, \ldots, n$ are transit providers. The little circles represent non-overlay users, and solid little circle represents overlay user.

Since the objectives of the ISP and overlay routing are not consistent, we model their interaction as a noncooperative routing game. From the ISP's viewpoint, a natural approach to traffic engineering involves controlling nonoverlay and overlay traffic as it pleases. Even though the ISP has the ability to control the overlay traffic routes, however, we find that this strategy cannot guarantee the existence of a Nash equilibrium of the routing game. Thus, network routing oscillation may occur such that the routing game never converges. We therefore propose a routing strategy for the ISP in which it controls only non-overlay traffic, and then plays a noncooperative game with the overlay applications. Under this strategy, at least one Nash equilibrium of the routing game always exists, which implies that the routing game is able to converge to one or more stable states. Exploring the structure of the Nash equilibrium, we find that the Nash equilibrium is not unique, but the distribution of traffic among all transit links is unique. We also prove that the proposed routing strategy guarantees convergence of the routing game in finite steps under any initialization conditions. Through numerical simulations, we demonstrate the convergence and efficiency of our strategy, and investigate the conditions under which the ISP can achieve minimum cost.

The rest of this paper is organized as follows. In Sect. 2, we introduce related research. In Sect. 3, we study the routing game between the ISP and overlay applications by using a game-theoretic model, and propose an ISP routing strategy that mitigates the negative impact of overlay routing. Then, in Sect. 4, the results from a set of numerical simulations are shown. Finally, conclusions are given in Sect. 5 .

\section{Related Work}

Related work falls into two categories: overlay networks and routing games in congested networks.

The earliest hypothesis regarding routing games in congested networks was the "first principle of user equilibrium" proposed in [7]. According to this principle, agents in a congested network choose their routes selfishly, a behavior that is captured by the Nash equilibrium of the underlying non- cooperative game. Devarajan [8] was the first to prove that a continuous flow, user-optimized network is a Nash equilibrium in a game with a continuum of pure strategies. In [6], Orda et al. considered a communication network shared by several selfish users in which each user seeks to optimize its performance by controlling the routing of its given flow demand. They investigated the Nash equilibria for such a noncooperative game and discussed the issue of convergence to an extent. Korilis et al. [9] then assumed that a network operator controls part of the network flow and is aware of the noncooperative behavior of the users. Focusing on the inefficiency of Nash equilibria, they proposed a Stackelberg routing strategy to achieve network optima from the standpoint of the network operator.

The current paper differs from the above research in that those studies examine properties that are common across general networks. However, we propose the more practical scenario of a multihoming network, and the routing game players are overlay routing traffic and the multihomed ISP. Moreover, ISP's cost in our study includes not only congestion cost but also the monetary cost from using transit links.

Extensive research on overlay routing has also been conducted, such as [10] and [3]. Such research has shown that overlay routing schemes are effective in solving some of the deficiencies in today's Internet. Resilient overlay networks (RON) [3] were the first wide-scale overlay implementation, and have been used as a testbed for several measurement studies. Investigations on overlay routing generally focus on the enhanced service that can be provided by overlay networks [11], [12]. Overlay path selection algorithms that attempt to provide quality-of-service-aware routing were studied in [12]. Since overlay networks rely heavily on active probing, their scalability and interaction with native networks have also been topics of interest [13]. The routing policy mismatch between overlay and ISP intradomain engineering was also examined in [5].

Unlike the above research, our focus here is on not only overlay routing but also the interaction between overlay routing and ISP inter-domain traffic engineering. We believe this issue must be addressed to improve inter-domain traffic service quality, as well as reduce ISP operational costs.

\section{Routing Game between ISP and Overlay Applica- tions}

\subsection{Network and Traffic Models}

In this section, we introduce the notation and mathematical models used throughout the remainder of the paper. For clarity, Table 1 lists most of the notation used in the model. Consider an ISP that connects to the Internet through $n$ links leased from transit providers. We denote the set of all transit links by $L$, the $i$ th link in $L$ by $l_{i}$, and the number of links in $L$ by $|L|(=n) . l_{i}$ 's capacity is expressed as $c_{i}$, and its price for transmitting traffic is $p_{i}$ per unit traffic. We assume that no two links in $L$ are identical in terms of both capacity and 
Table 1 Notation used in mathematical model.

\begin{tabular}{|ll|}
\hline$L:$ & Set of all transit links \\
$c_{i}:$ & Link capacity of $l_{i}$ \\
$p_{i}:$ & Price per unit traffic for using $l_{i}$ \\
$\gamma:$ & Multihomed ISP's sensitivity to monetary cost. It is a weight \\
& factor to adjust the relative importance of monetary cost and \\
& latency cost \\
$f_{i}^{o l}:$ & Overlay traffic volume on $l_{i}$ \\
$f_{i}^{n o l}:$ & Non-overlay traffic volume on $l_{i}$ \\
$f^{o l}:$ & Total overlay traffic volume \\
$f^{n o l}:$ & Total non-overlay traffic volume \\
$f:$ & Total traffic volume \\
$\beta_{i}:$ & The marginal cost of ISP on $l_{i}$, which is the derivative of \\
& ISP's cost on $l_{i}$ w.r.t. the non-overlay traffic volume $f_{i}^{n o l}$ \\
$A_{i}$ & The latency of $l_{i}$ \\
$U_{i}$ & ISP's cost on $l_{i}$ \\
$U$ & ISP's total cost of each link in $L$ \\
$V$ & The potential function of overlay applications \\
$L^{O}:$ & Set of links with only overlay traffic \\
$L^{N}:$ & Set of links with only non-overlay traffic \\
$L^{B}:$ & Set of links with both non-overlay and overlay traffic \\
$L^{-}(t):$ & Set of links from which traffic is removed at step $t$ \\
$L^{+}(t):$ & Set of links that receive traffic at step $t$ \\
$\Delta(t):$ & Total traffic movement between $L^{-}(t)$ and $L^{+}(t)$ at step $t$ \\
$\delta_{i}(t):$ & Difference in traffic volume on $l_{i}$ before and after step $t$ \\
\hline
\end{tabular}

price. This network can be conceptualized as a model with parallel links, as shown in Fig. 1.

Network traffic consists of non-overlay and overlay routing traffic. The routing of non-overlay traffic is controlled by only the ISP, whereas overlay traffic can be also controlled by overlay applications. If we denote non-overlay and overlay traffic volumes as $f^{n o l}$ and $f^{o l}$, respectively, then the total traffic volume on the network is $f=f^{n o l}+f^{o l}$. Furthermore, two assumptions are used in this paper:

Assumption 1. Traffic flow can be arbitrarily split among any number of links (splittable flow).

Assumption 2. The ISP can distinguish between overlay traffic and non-overlay traffic, and can control both of them.

In fact, the multihomed ISP can split the traffic to various upstream transit ISPs with intelligent routing control technologies as in [1], [2], and [14]. ISP can also identify overlay traffic by traffic flow analysis and deep packet inspection, and then the ISP can delay overlay traffic flows on certain paths with traffic shaping technology to control the overlay applications' routing choice indirectly.

Under these two assumptions, the ISP and overlay applications can adjust the traffic volume through each link according to their individual preferences. If we define the volumes of non-overlay and overlay routing traffic on link $l_{i}$ as $f_{i}^{\text {nol }}$ and $f_{i}^{o l}$, respectively, then the total traffic volume on $l_{i}$ is $f_{i}=f_{i}^{\text {nol }}+f_{i}^{\text {ol }}$. The total ISP's cost is composed of two costs in this paper: monetary and latency. To transmit data through transit links, the ISP must pay the transit providers. Moreover, when making routing decisions, the ISP needs to consider not only monetary cost but also the quality of service. We assume that the transmission delay of each link is represented by an $M / M / 1$ model, and that the ISP uses the average latency of each link as the congestion metric. Here, we follow the latency cost model presented in [15] in which the latency of $l_{i}$ and its associated cost are given by $\frac{1}{c_{i}-f_{i}}$ and $\frac{f_{i}}{c_{i}-f_{i}}$, respectively. We then formulate the ISP's cost for $l_{i}$ as the weighted sum of the monetary and latency costs:

$$
U_{i}=\frac{\left(f_{i}^{n o l}+f_{i}^{o l}\right)}{c_{i}-f_{i}^{n o l}-f_{i}^{o l}}+\gamma\left(f_{i}^{n o l}+f_{i}^{o l}\right) p_{i},
$$

where $\gamma(\geq 0)$ is the weighting factor of the monetary cost. In fact, $\gamma$ can also be considered as the ISP's sensitivity to monetary cost. Then the ISP's total cost is the sum of cost of each link:

$$
U=\sum_{l_{i} \in L} U_{i}
$$

and the ISP's objective is thus to solve the following optimization problem:

$$
\begin{aligned}
& \left(f_{1}, \ldots, f_{n}\right)=\arg \min U \\
& \text { subject to } f_{i}^{\text {nol }} \geq 0, f_{i}^{o l} \geq 0, f_{i}<c_{i}, \sum_{l_{i} \in L} f_{i}^{\text {nol }}=f^{\text {nol }} .
\end{aligned}
$$

Various overlay applications may exist in the network, such as P2P, service overlay network and CDN. Most of the overlay traffic behaves selfishly without regard to the ISP's cost. In the early days of $\mathrm{P} 2 \mathrm{P}$ file sharing networks, one peer selects neighbors randomly. But today, extensive research has been done on neighbor selection, and selecting neighbors with the lowest latency has become mainstream [16], [17]. Service overlay networks such as Detour [10] and RON [3] also take latency as the most important performance metric. One of the main objectives of the CDN networks such as Akamai is to minimize end-to-end latency by choosing alternative paths [4]. We can see that although various overlay applications exist, most of them choose paths according to latency. Therefore, in this paper, we assume that overlay applications utilize latency as an indicator of link performance, and thus they always choose the link with minimum latency. According to [18], the interaction of various overlay traffics can be seen as a Wardrop game, and the equilibria of Wardrop game cannot be expressed as a tractable optimization problem. In [18], the authors propose a method to characterize the equilibria of Wardrop game by constructing a potential function. The idea is to construct a real-valued function, which is defined on the set of possible outcomes of the game, such that the equilibria of the game are precisely the optima of this function. The potential function must enable the application of optimization theory. A potential function of the overlay routing game in our work can be constructed as follows:

$$
V=\sum_{l_{i} \in L} \int_{0}^{f_{i}^{o l}} \frac{1}{c_{i}-f_{i}^{n o l}-x} d x .
$$

With necessary constraints, we have the following convex 
non-linear constrained optimization problem.

$$
\begin{aligned}
& \left(f_{1}^{o l}, \ldots, f_{n}^{o l}\right)=\arg \min V, \\
& \text { subject to } f_{i}^{\text {nol }}+x<c_{i}, \sum_{l_{i} \in L} f_{i}^{o l}=f^{o l} .
\end{aligned}
$$

By applying the convex optimization theory, we can find the solution of the optimization problem is that every route with non-zero overlay traffic has the same latency, which is just the characterization of the overlay routing game equilibria. In other words, the equilibria of the overlay routing game are precisely the solution that minimizes the potential function with constraints. By introducing the potential function, we can solve optimization problem (5) to obtain the overlay routing game equilibra, instead of solving the origin problem directly. In fact, this method is widely used in game theory studies [18]-[20]. Given the objective function of the ISP and the potential function of overlay applications, we are now ready to define the routing game in the next section.

\subsection{Nash Routing Game}

Although the optimal decision of each player in Eqs. (3) and (5) clearly depends on the decisions of the other players, since their behaviors are selfish, we are faced with a noncooperative game. If the ISP and overlay routing strategies are flow configurations denoted by $\left(f_{1}, \ldots, f_{n},\right)$ and $\left(f_{1}^{o l}, \ldots, f_{n}^{o l}\right)$, respectively, then we obtain the definition of a Nash equilibrium for this routing game.

Definition 1. A strategy profile $\left\{\left(f_{1}, \ldots, f_{n}\right),\left(f_{1}^{o l}, \ldots, f_{n}^{o l}\right)\right\}$ is a Nash equilibrium for both the ISP and overlay routing if the following conditions hold:

$$
\begin{aligned}
& U\left\{\left(f_{1}^{*}, \ldots, f_{n}^{*}\right),\left(f_{1}^{o l^{*}}, \ldots, f_{n}^{o l^{*}}\right)\right\} \\
\leq & U\left\{\left(f_{1}, \ldots, f_{n}\right),\left(f_{1}^{o l^{*}}, \ldots, f_{n}^{o l^{*}}\right)\right\}, \\
& V\left\{\left(f_{1}^{*}, \ldots, f_{n}^{*}\right),\left(f_{1}^{o l^{*}}, \ldots, f_{n}^{o l^{*}}\right)\right\} \\
\leq & V\left\{\left(f_{1}^{*}, \ldots, f_{n}^{*}\right),\left(f_{1}^{o l}, \ldots, f_{n}^{o l}\right)\right\},
\end{aligned}
$$

in which $U$ is ISP's total cost, and $V$ is overlay applications' total performance cost. In fact, we can imagine that in the routing game, the ISP and overlay applications make routing choice in an alternate way as in [21]. ISP would execute the traffic engineering process to reallocate traffic periodically. After ISP's traffic engineering, overlay applications would react by reallocating overlay traffic, and in the ISP's next traffic engineering period, ISP will react to overlay traffic by reallocating traffic again.

\subsection{ISP's Routing Strategies}

The ISP would naturally like to simultaneously control overlay and non-overlay traffic in order to minimize its total cost. Furthermore, the minimum cost appears to be realized under such a strategy. However, the existence of a Nash equilibrium is not guaranteed by employing the strategy, and it may

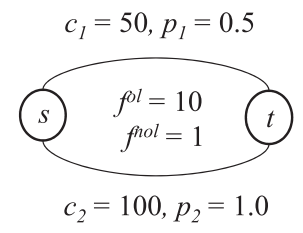

Fig. 2 A simple example in which Nash equilibrium does not exist.

cause persistent route oscillation. An example in which the game never converges is straightforward to set up. Assume a network has two transit links (Fig. 2): $l_{1}$ with $c_{1}=50$ and $p_{1}=0.5$, and $l_{2}$ with $c_{2}=100$ and $p_{2}=1.0$. In addition, let the non-overlay and overlay traffic volumes be 1 and 10, respectively. Then, from Eq. (3), the ISP will force all traffic to pass through $l_{1}$, whereas the overlay routing will reroute the overlay traffic through $l_{2}$ from Eq. (5). Thus, a Nash equilibrium does not exists, and route oscillation cannot be avoided. To ensure that the routing game converges to a stable state, we propose a routing strategy in which the ISP controls only non-overlay traffic, and plays a noncooperative game with the overlay applications. Then, the ISP's objective function in Eq. (3) can be rewritten as

$$
\begin{aligned}
& \left(f_{1}^{n o l}, \ldots, f_{n}^{n o l}\right)=\arg \min U \\
& \quad \text { subject to } f_{i}^{n o l} \geq 0, f_{i}^{o l} \geq 0, f_{i}<c_{i}, \sum_{l_{i} \in L} f_{i}^{n o l}=f^{n o l} .
\end{aligned}
$$

In fact, the ISP which behaves as above just satisfies the definition of a "group user" in [22], who has a large volume traffic to be delivered by splitting the traffic to multiple paths to minimize the total cost of each path. The overlay applications in our work just satisfy the definition of "individual user" in [22], whose objective is always to choose the lowest cost path. The authors of [22] call the equilibria of a game played by group users and individual users as mixed equilibria, and they also proved that, if the objective functions of group users are non-negative, convex and continuously differentiable, and the objective functions of individual users are non-negative and continuous, then there exists a mixed equilibrium. Since the objective function of the ISP and potential function of overlay applications in our work satisfy the requirements above, we can obtain the following proposition:

Proposition 1. A Nash equilibrium always exists for the routing game stated in this paper.

Two properties of the Nash solution are important: uniqueness and convergence. Before discussing the two properties, we first explore the Nash solution structure, and obtain some results which can be used in the proof of uniqueness and convergence. Now, let us focus on the structure of the Nash equilibria. We begin by introducing some notation. Suppose that all links fall into the following four categories at a Nash equilibrium: the set of links with only non-overlay traffic denoted $L^{N}$, the set of links with only 


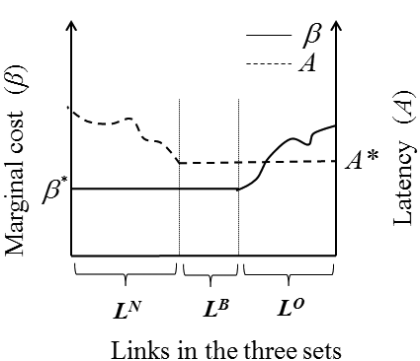

(a)

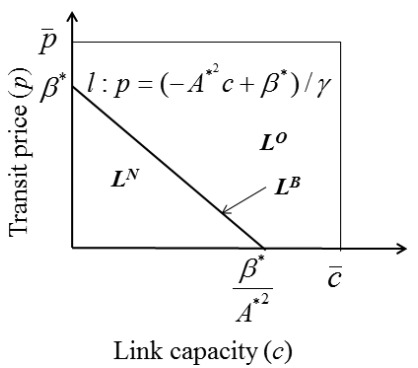

(b)

Fig. 3 Nash equilibrium structure.

overlay traffic denoted $L^{O}$, the set of links with both nonoverlay and overlay traffic denoted $L^{B}$, and the set of links without traffic denoted $L^{E}$. Then, to solve the constrained optimization problem in Eq. (7), if we examine the KKT conditions, we find that $\left(f_{1}^{n o l^{*}}, \ldots, f_{n}^{n o l^{*}}\right)$ are optimal for the ISP if and only if there exists a Lagrange multiplier $\beta^{*}$ such that for every $l_{i} \in L^{N} \cup L^{B}$

$$
\begin{aligned}
& \beta^{*}=\left.\frac{\partial U}{\partial f_{i}^{n o l}}\right|_{f_{i}^{n o l}=f_{i}^{n o l^{*}}}=\frac{c_{i}}{\left(c_{i}-f_{i}^{n o l^{*}}-f_{i}^{o l}\right)^{2}}+\gamma p_{i}, \text { if } f_{i}^{n o l^{*}}>0, \\
& \beta^{*} \leq\left.\frac{\partial U}{\partial f_{i}^{n o l}}\right|_{f_{i}^{n o l}=0}=\frac{1}{c_{i}}+\gamma p_{i}, \quad \text { if } \quad f_{i}^{n o l^{*}}=0,
\end{aligned}
$$

where $U$ is ISP's objective function in Eq. (3). In actuality, $\beta_{i}=\frac{\partial U}{\partial f_{i}^{\text {nol }}}=\frac{c_{i}}{\left(c_{i}-f_{i}\right)^{2}}+\gamma p_{i}$ can be viewed as the ISP's marginal cost for link $l_{i}$, which measures the cost increase if the ISP adds an addition unit of traffic to $l_{i}$. Based on the concept of marginal cost, the above conditions are easy to understand. Given two links with positive non-overlay traffic volume, the marginal costs must be the same at Nash equilibria. Otherwise, the ISP will keep moving traffic from the link with larger marginal cost to the link with lower marginal cost. At Nash equilibria, if the marginal cost of one link is larger than another link with positive non-overlay traffic volume, the non-overlay traffic passing through the larger marginal cost link must be zero for the same reason as the former case.

Similarly, for overlay routing, $\left(f_{1}^{o l^{*}}, \ldots, f_{n}^{o l^{*}}\right)$ are optimal if and only if there exists $A^{*}>0$ such that for every link $l_{j} \in L^{O} \cup L^{B}$

$$
\begin{aligned}
& A^{*}=\left.\frac{\partial V}{\partial f_{i}^{o l}}\right|_{f_{i}^{o l}=f_{i}^{o l^{*}}}=\frac{1}{c_{j}-f_{j}^{n o l}-f_{j}^{o l^{*}}}, \quad \text { if } f_{j}^{o l^{*}}>0, \\
& A^{*} \leq\left.\frac{\partial V}{\partial f_{i}^{o l}}\right|_{f_{i}^{o l}=0}=\frac{1}{c_{j}-f_{j}^{n o l}}, \quad \text { if } \quad f_{j}^{o *^{*}}=0,
\end{aligned}
$$

where $V$ is the potential function in Eq. (5). The above conditions imply that at Nash equilibria, all links with positive overlay traffic volume must have identical latency. If the latency of a specific link is larger than another link with positive overlay traffic volume, then the overlay traffic volume on the link with larger latency must be zero.
From Eqs. (8) and (9), we can also conclude that $\forall l_{i} \in L^{B}$, $p_{i}=\left(-A^{* 2} c_{i}+\beta^{*}\right) / \gamma$.

The above conditions can be intuitively represented, as shown in Fig. 3 (a). In this diagram, we show marginal costs and latencies for three of the link sets ( $L^{E}$ is omitted for the sake of clarity). Here, $\bar{c}$ and $\bar{p}$ are the maximal link capacity transit price, respectively. The links in $L^{N}$ clearly have an identical marginal $\operatorname{cost} \beta^{*}$, which is lower than the marginal costs of links in $L^{O}$. Moreover, the links in $L^{O}$ have identical latency $A^{*}$, and $A^{*}$ is lower than the latency of links in $L^{N}$. The links in $L^{B}$ have marginal cost $\beta^{*}$ and latency $A^{*}$. Link set classification is determined by the link capacities, monetary costs, and traffic volumes. The relations among the variables are shown in Fig. 3 (b). Each link is represented by a point with a specific link capacity and transit price. At a Nash equilibrium, the line given by $p=\left(-A^{* 2} c+\beta^{*}\right) / \gamma$ splits the region into two. The links below this line are in $L^{N}$. Explicitly, because $\forall l_{i} \in L^{N}, p_{i}=\left(-A_{i}^{2} c_{i}+\beta^{*}\right) / \gamma$ with $A_{i}>A^{*}$, the point $\left(c_{i}, p_{i}\right)$ is below the line given by $p$. Similarly, the links above the line are in $L^{O}$, and the links on the line are in $L^{B}$. Note that in Fig. 3 (b), we take the traffic volume as constant and only discuss the link capacities and monetary costs. We will show the effect of traffic volume with numerical experiment in Sect. 4. Given the above structure of a Nash equilibrium, we can prove the following property:

Lemma 1. The link classification scheme at a Nash equilibrium is unique.

The proof of Lemma 1 is given as an appendix. Proposition 2 then follows immediately from Lemma 1 and Eqs. (8) and (9):

Proposition 2. At a Nash equilibrium, $\forall l_{i} \in L^{N}, f_{i}^{n o l^{*}}$ is unique; $\forall l_{j} \in L^{O}, f_{j}^{o l^{*}}$ is unique; and $\forall l_{k} \in L^{B}$, the total traffic volume $f_{k}^{n o l^{*}}+f_{k}^{o l^{*}}$ is unique.

We denote the ISP's cost at a Nash equilibrium as $U^{\text {Nash }}$, and the cost immediately after ISP traffic engineering, which adjusts both non-overlay and overlay traffic, as $U^{\text {Min }}$. Clearly, $U^{\text {Nash }} \geq U^{\text {Min }}$; however, since overlay routing timescales to adjust traffic are always considerably smaller than those of the ISP, the majority of the time is spent in a routing status preferable to the overlay routing. We denote the ISP's cost when the overlay routing has full 
traffic control as $U^{M a x}$. Based on Eqs. (7), (5), (8), and (9), the relations among the three costs can be summarized as.

Corollary 1. $U^{\text {Min }} \leq U^{\text {Nash }} \leq U^{\text {Max }}$.

Since realizing $U^{\text {Min }}$ is nontrivial in practice, we claim that the ISP can avoid routing oscillation by using the proposed strategy at the relatively low cost of $U^{\text {Nash }}$. The situations in which $U^{\text {Nash }}$ can attain $U^{\text {Min }}$ are of interest here, and so we discuss this issue quantitatively in the numerical simulations.

\subsection{Convergence of the Routing Game}

In this section, we investigate the convergence of the routing game. Such an investigation is important because a routing game may not always converge even if a Nash equilibrium exists. Liu et al. [5] showed an example in which the routing game convergence is dependent on its initial state. Here, we assume that the ISP and overlay routing play the game in turn, and let a step of the game denote the best response of the current player to the previous step. At each step, one of the players reallocates its flow such that its individual cost is minimized. During the routing game, if the difference in traffic volume on each link between two consecutive steps is less than a small threshold, we can say that the routing game has converged. Under these conditions, we obtain the following result:

Proposition 3. The routing game can always converge independently of the initial state.

Proof. First, we introduce some notation. Suppose at step $t$, either the ISP or overlay applications reallocates the traffic on some links. Then, we denote the link set from which traffic is removed at $t$ as $L^{-}(t)$, the link set that receive traffic at step $t$ as $L^{+}(t)$, the difference in traffic volume on $l_{i}$ before and after step $t$ as $\delta_{i}(t)$, and the total traffic movement as $\Delta(t)$.

Suppose $t \geq 3$, and at step $t$, the overlay applications are reallocating traffic. Overlay applications remove $\Delta(t)$ overlay traffic from the links in $L^{-}(t)$ and place this on those in $L^{+}(t)$. This implies that before step $t$, the latencies of links in $L^{-}(t)$ were larger than those in $L^{+}(t)$. After step $t$, the marginal costs of links in $L^{+}(t)$ will be larger than those in $L^{-}(t)$, since prior to the reallocation at step $t$, the traffic allocation was preferable to the ISP according to marginal costs as in Eq. (7). Therefore, in step $t+1$, the ISP attempts to move the non-overlay traffic from $L^{+}(t)$ to $L^{-}(t)$.

Assume after step $t, \forall l_{i}$ in $L^{+}(t)$, the traffic added is $\delta_{i}(t)$. If $\forall l_{i}$ in $L^{+}(t)$, the non-overlay traffic on $l_{i}$ is $\geq \delta_{i}(t)$, then at step $t+1$, the ISP will remove $\delta_{i}(t)$ non-overlay traffic from $l_{i}$, and it follows that $\Delta(t+1)=\Delta(t)$.

If after step $t$, the non-overlay traffic on a certain link $l_{j} \in L^{+}(t)$ is $<\delta_{j}(t)$, then at step $t+1$, ISP will remove all of the non-overlay traffic on $l_{j}$. Now let us discuss the relation between $\Delta(t+1)$ and $\Delta(t)$ in such a case. Assume $\Delta(t+1) \geq$ $\Delta(t)$. Since $\delta_{j}(t+1)<\delta_{j}(t)$, then $\forall l_{k} \in\left(L^{+}(t)-l_{j}\right)$, there must be $\delta_{k}(t+1)>\delta_{k}(t)$. Then, after step $t+1$, the marginal costs of the links in $L^{+}(t+1)$ are equal to the marginal costs on $L^{+}(t-1)$ before step $t$. In contrast, $\forall l_{k} \in\left(L^{+}(t)-l_{j}\right)$, the marginal cost of $l_{k}$ is less than the marginal costs of the links in $L^{+}(t-1)$ before step $t$. It follows that, after step $t+1, \forall l_{k} \in\left(L^{+}(t)-l_{j}\right)$, the marginal cost of $l_{k}$ is less than the marginal costs of the links in $L^{+}(t+1)$, which contradicts Eq. (7). Thus, we conclude that $\Delta(t+1)<\Delta(t)$.

Denote $f^{+}(t)$ as the total traffic volume of links in $L^{+}(t)$, and $f^{-}(t)$ as the total traffic volume of links in $L^{-}(t)$. Then we have the following equations:

$$
\begin{aligned}
& f^{+}(t+2)=f^{+}(t)-\Delta(t)+\Delta(t+1) \leq f^{+}(t), \\
& f^{-}(t+2)=f^{-}(t)+\Delta(t)-\Delta(t+1) \geq f^{-}(t) .
\end{aligned}
$$

Since the number of links is finite and the traffic volume is bounded, $f^{+}(t)$ and $f^{-}(t)$ converge as $t \rightarrow \infty$, which obviously implies the convergence of the routing game.

Given Proposition 3, the ISP has two ways to apply the routing strategy. If the ISP has full knowledge of the overlay traffic, it can compute the non-overlay traffic allocation $\left(\ldots, f_{i}^{n o l^{*}}, \ldots\right)$ at a Nash equilibrium offline, and allocate the non-overlay traffic a priori. If the ISP has insufficient knowledge of the overlay traffic, it can play the routing game online. In the online scheme, ISP controls only non-overlay traffic, and adjusts non-overlay traffic to minimize the total cost periodically, until the traffic allocation is preferable and not re-allocated by overlay applications any more. The time scale between ISP's consecutive adjusting should be long enough for overlay applications to finish overlay traffic adjusting.

\section{Numerical Simulations}

To give intuitive examples of our results, we present several numerical simulations in this section. In all simulations, we assume a network composed of 8 links with the properties listed in Table 2. In the first example, we show the convergence and the solution structure of the routing game. We set the total traffic volume $f=120$, and ISP's sensitivity to monetary cost $\gamma=1$. Suppose that the non-overlay and overlay traffic volumes are identical. The initial traffic distribution is set such that each link's volume is proportional to its capacity. The traffic distribution in each step of the simulation is shown in Fig. 4. After fewer than 15 steps, the traffic volume on each link becomes stable. We also show the marginal costs and latencies of all the links in Fig. 5. According to the properties of Nash equilibrium, link 1, 5, 6, 7 belong to $L^{N}$, since they have identical marginal cost, and link 2, 4,8 belong to $L^{O}$, since they have identical latency.

Table 2 Link properties.

\begin{tabular}{lllllllll}
\hline Link & 1 & 2 & 3 & 4 & 5 & 6 & 7 & 8 \\
\hline Capacity & 20 & 30 & 20 & 25 & 25 & 30 & 15 & 35 \\
Monetary cost & 0.2 & 0.4 & 0.4 & 0.5 & 0.3 & 0.2 & 0.2 & 0.6 \\
\hline
\end{tabular}


Link 3 belongs to $L^{B}$, because it has identical marginal cost with the links in $L^{N}$, and identical latency with the links in $L^{O}$.

In the second experiment, we compare the ISP's costs $U^{\text {Min }}, U^{\text {Nash }}$, and $U^{\text {Max }}$ in three cases: $\gamma=0.5, \gamma=1.0$, and $\gamma=1.5$. Figure 6 shows that the relations among $U^{M i n}$, $U^{\text {Nash }}$, and $U^{\text {Max }}$ are consistent with Corollary 1 . Hence, the ISP's cost under the proposed strategy is always between those in the worst and best cases. We can find from the figures that when the traffic load is light, $U^{\text {Nash }}, U^{\text {Min }}$ and $U^{M a x}$ are significantly different from each other. With the traffic volume increasing, the differences among them become smaller. When the traffic load is quite heavy, the three costs also become similar to each other. In fact, the network with extremely heavy traffic load is not common in practice. In general, we should consider the network with moderate traffic load, and then the proposal in this paper makes sense. Note that even for a network with extremely heavy

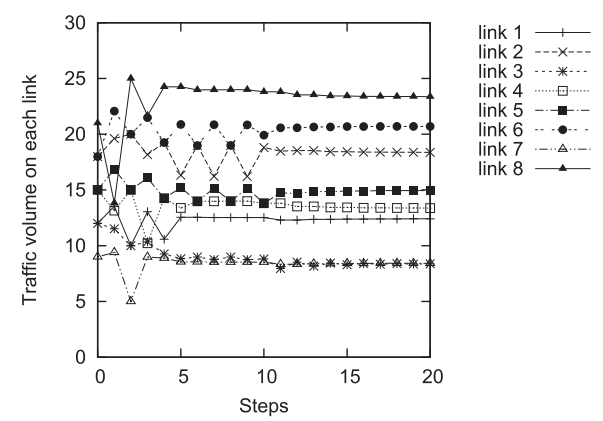

Fig. 4 Convergence of routing game.

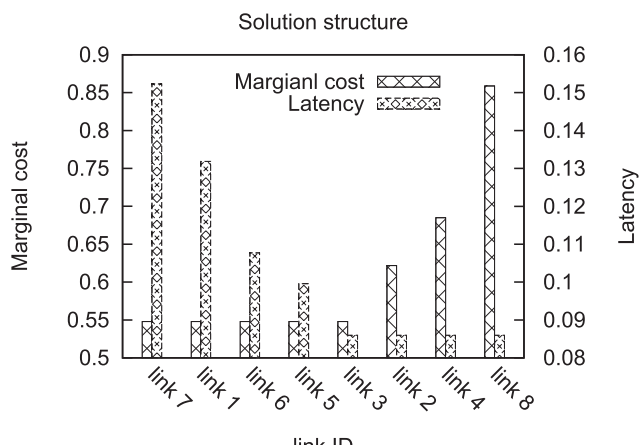

Fig. 5 Solution structure of the routing game. traffic load, as we did not make assumption of the unit of cost, the absolute differences of the three costs would still be significant if the unit of cost is quite large (e.g. thousands of dollars). Also note that in the experiments, the value $\gamma$ only affects the absolute values of ISP's costs, but does not affect the relation of $U^{\text {Min }}, U^{\text {Nash }}$, and $U^{\text {Max }}$. From Fig. 6 we can see that, as the total traffic volume increases, the relative differences among $U^{\text {Min }}, U^{\text {Nash }}$, and $U^{\text {Max }}$ become smaller. Hence, the ISP's cost under the proposed strategy approaches the minimum cost. The situations in which the ISP's cost under the proposed strategy can actually attain the minimum cost are of interest. We investigate this in the next simulation.

We find that the non-overlay/total traffic ratio and the ISP's sensitivity to monetary cost $\gamma$ may have a considerable impact on whether the ISP can realize the minimum cost. We denote the proportion of non-overlay traffic by $r$. From the simulation, we find that, if the total traffic exceeds a given volume, a value $\theta(0<\theta<1)$ can always be found such that, if $r \geq \theta$, then the ISP can realize the minimum cost under the proposed strategy. Explicitly, the minimum cost can be achieved if and only if $L^{O}=\emptyset$ and all of the overlay traffic is routed on the links in $L^{B} \cup L^{N}$. From Fig. 7, $\theta$ decreases with respect to the total traffic volume. This decrease implies that the ISP could control a small traffic volume to negate the difference between the cost under the proposed strategy and the minimum cost under a heavier traffic load. We also find that $\gamma$ has a considerable effect on $\theta$. In this simulation, an increase in $\gamma$ causes an increase in $\theta$ since monetary costs are not consistent with latency costs. Specifically, the objectives of overlay applications and ISP's traffic engineering are closer with a smaller $\gamma$, which suggests that the ISP can realize the minimum cost "easier", and vice

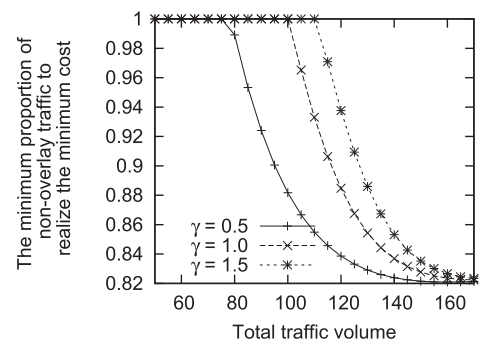

Fig. 7 Minimum proportion of non-overlay traffic to obtain minimum cost.

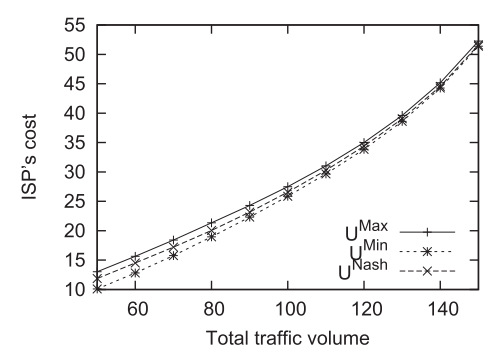

(a) $\gamma=0.5$

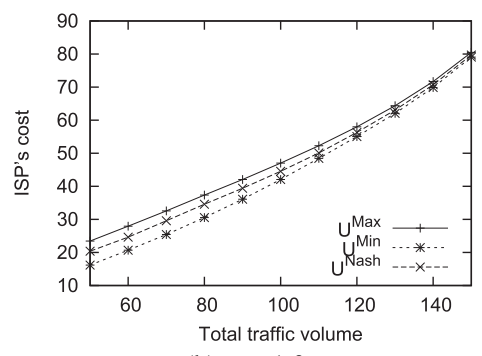

(b) $\gamma=1.0$

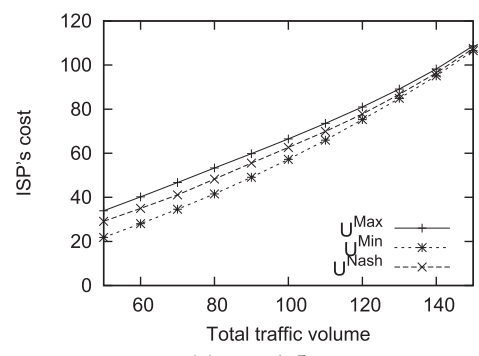

(c) $\gamma=1.5$

Fig. 6 ISP's total costs for various cases. 
versa.

\section{Conclusion}

We have studied the interaction between overlay traffic and a multihomed ISP's routing decisions by using game theory. We showed that if the ISP does not differentiate between overlay and non-overlay traffic, then permanent routing oscillation may occur. By proposing a routing strategy under which the ISP controls only non-overlay traffic, we proved that a Nash equilibrium of the routing game always exists. Studying the structure of the Nash equilibria, we were able to show the uniqueness of traffic allocation at an equilibrium. We then proved that the routing game when using our strategy always converges to a Nash equilibrium. These properties ensure that the traffic routing will eventually achieve a stable state in all cases. At the same time, the ISP can keep the overall cost relatively low. By conducting numerical simulations, we gave concrete examples of the routing game results, and discussed the conditions under which the ISP can realize the minimum cost under the proposed strategy.

In the future, we will consider more kinds of overlay applications with various performance indicators. We will also investigate the cooperation of ISP' traffic engineering and overlay applications.

\section{References}

[1] D.K. Goldenberg, L. Qiu, H. Xie, Y.R. Yang, and Y. Zhang, "Optimizing cost and performance for multihoming," Proc. SIGCOMM 2004, pp.79-92, Aug. 2004.

[2] R. Dai, D.O. Stahl, and A.B. Whinston, "The economics of smart routing and quality of service," Proc. International Workshop on Networked Group Communications 2003, pp.318-331, 2003.

[3] D. Anderson, H. Balakrishnan, M.F. Kaashoek, and R. Morris, "Resillient overlay networks," Proc. Eighteenth ACM Symposium on Operating Systems Principles, pp.131-145, Oct. 2001.

[4] Akamai, "http://www.akamai.com."

[5] Y. Liu, H. Zhang, W. Gong, and D. Towsley, "On the interaction between overlay routing and underlay routing," Proc. IEEE INFOCOM 2005, pp.2543-2553, April 2005.

[6] A. Orda, R. Rom, and N. Shimkin, "Competitive routing in multiuser communication networks," IEEE/ACM Trans. Networking, vol.1, no.5, pp.510-521, Oct. 1993.

[7] J.G. Wardrop, "Some theoretical aspects of road traffic research," Proc. Institution of Civil Engineers 1952, Part II, pp.352-362, 1952.

[8] S. Devarajan, "A note of network equilibrium and noncooperative games," Transportation Research Part B, vol.15, no.6, pp.421-426, Dec. 1981.

[9] Y.A. Korilis, A.A. Lazar, and A. Orda, "Achieving network optima using stackelberg routing strategies," IEEE/ACM Trans. Netw., vol.5, no.1, pp.161-173, Feb. 1997.

[10] S. Savage, T. Anderson, A. Aggarwal, D. Becker, N. Cardwell, A. Collins, E. Hoffman, J. Snell, A. Vahdat, G. Voelker, and J. Zahorjan, "Detour: A case for informed internet routing and transport," IEEE Micro, vol.19, no.1, pp.50-59, Jan. 1999.

[11] L. Subramanian, I. Stoica, H. Balakrishnan, and R. Katz, "OverQoS: Offering Internet QoS using overlays," ACM SIGCOMM Computer Communication Review, vol.33, no.1, pp.11-16, Jan. 2002.

[12] Z. Li and P. Mohapatra, "QRON: QoS-aware routing in overlay networks," IEEE J. Sel. Areas Commun., vol.22, no.1, pp.29-40, Sept.
2006.

[13] A. Nakao, "A routing underlay for overlay networks," Proc. ACM SIGCOMM 2003, pp.11-18, Aug. 2003.

[14] Y. Liu and A.L.N. Reddy, "Multihoming route control among a group of multihomed stub networks," Comput. Commun., vol.30, no.17, pp.3335-3345, Nov. 2007.

[15] S.C.M. Lee, J.W.J. Jiang, D.M. Chiu, and J.C.S. Lui, "Interaction of ISPs: Distributed Resource Allocation and Revenue Maximization," IEEE Trans. Parallel Distrib. Syst., vol.19, no.2, pp.204-218, Feb. 2008.

[16] Y. Jiang and J. Wu, "Employing QoS driven neighbor selection for heterogeneous peer-to-peer streaming," Proc. IEEE ICC 2011, pp.16, July 2011.

[17] R.L. Pereira, "On the impact of P2P file sharing traffic restrictions on user perceived performance," Proc. ICOIN 2008, pp.1-5, Jan. 2008.

[18] M. Beckmann, C.B. McGuire, and C.B. Winsten, Studies in the Economics of Transportation, Yale University Press, 1956.

[19] T. Roughgarden and É. Tardos, "How Bad is Selfish Routing?," J. ACM, vol.49, no.2, pp.236-259, March 2002.

[20] T. Roughgarden, "Potential functions and the inefficiency of equilibria," Proc. International Congress of Mathematicians 2006, pp.1071-1094, Aug. 2006.

[21] L. Qiu, Y.R. Yang, Y. Zhang, and S. Shenker, "On selfish routing in Internet-like environments," Proc. ACM SIGCOMM 2003, pp.151162, Aug. 2003.

[22] T. Boulogne, E. Altman, H. Kameda, and O. Pourtallier, "Mixed equilibrium (ME) for multiclass routing games," IEEE Trans. Autom. Control, vol.47, no.6, pp.903-916, June 2002.

\section{Appendix: Proof of Lemma 1}

Proof. Proof by contradiction. Assume that two different link classification schemes exist at a Nash equilibrium. One scheme is expressed by the sets $L^{N}, L^{O}$, and $L^{B}$, and the other by $\tilde{L}^{N}, \tilde{L}^{O}$, and $\tilde{L}^{B}$. Furthermore, the lines that split the domains under the two schemes are $l$ and $\tilde{l}$. Four types of relative positions then exist for $l$ and $\tilde{l}$, as shown in Fig. A. 1 . We outline the idea of the proof as follows.

For the case shown in Fig. A. 1 (a) with $\tilde{\beta}^{*}<\beta^{*}$, we see that the links in $\tilde{L}^{N} \cup \tilde{L}^{B}$ form a proper subset of $L^{N} \cup L^{B}$. Therefore, $\tilde{\beta}^{*}$ must be greater than $\beta^{*}$, which contradicts the original condition. Hence, this case is impossible.

For the case shown in Fig. A. 1 (a) with $\tilde{\beta}^{*}>\beta^{*}$, we see that the links in $L^{N} \cup L^{B}$ form a proper subset of $\tilde{L}^{N} \cup \tilde{L}^{B}$. Therefore $\tilde{\beta}^{*}$ must be less than $\beta^{*}$, which again contradicts the original condition. Hence, this case is also impossible.

For the case shown in Fig. A. 1 (b) with $\tilde{\beta}^{*}<\beta^{*}$, we see directly from the figure that in the second link classification scheme $\tilde{A}^{*}<A^{*}$. In addition, $\forall l_{i} \in L^{N} \cup \tilde{L}^{N}, \tilde{A}_{i}<A_{i}$ (because the slope of the line connecting $\left(c_{i}, p_{i}\right)$ and $\left(\tilde{\beta}^{*}, 0\right)$ is shallower than that of the line connecting $\left(c_{i}, p_{i}\right)$ and $\left.\left(\beta^{*}, 0\right)\right)$. This implies that all links have lower latencies in the second classification scheme than in the first, which cannot happen because the total traffic volume and all link capacities are constant. Hence, this case is impossible.

For the case shown in Fig. A. 1 (b) with $\tilde{\beta}^{*}>\beta^{*}$, we see directly from the figure that in the second link classification scheme $\tilde{A}^{*}>A^{*}$. In addition, $\forall l_{i} \in L^{N} \cup \tilde{L}^{N}, \tilde{A}_{i}>A_{i}$ (because the slope of the line connecting $\left(c_{i}, p_{i}\right)$ and $\left(\tilde{\beta}^{*}, 0\right)$ is steeper than that of the line connecting $\left(c_{i}, p_{i}\right)$ and $\left.\left(\beta^{*}, 0\right)\right)$. 


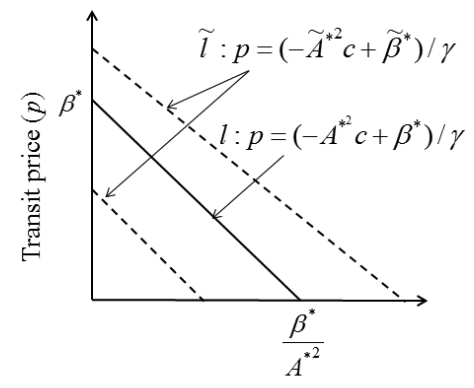

Link capacity $(c)$

(a)

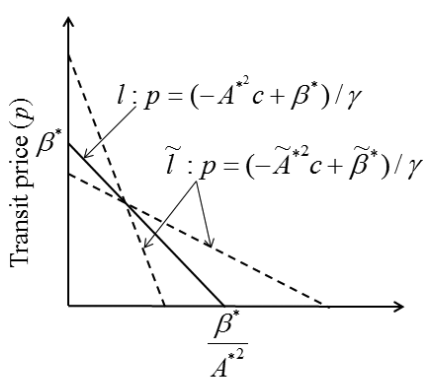

Link capacity $(c)$

(b)

Fig. A. 1 Four cases of the two link classification schemes.

This implies that all links have higher latencies in the second classification scheme than in the first, which again cannot happen because the total traffic volume and all link capacities are constant. Hence, this case is impossible. Thus, the link classification scheme is unique.

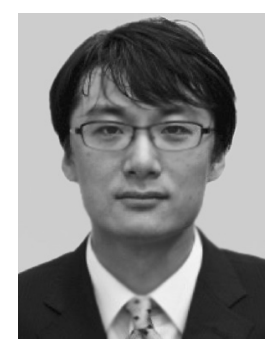

Xun Shao received his B.E. degree from Civil Aviation University of China, in 2005, and his M.E. degree from Beijing Jiaotong University, China, in 2008. His research interests include Internet structure and overlay routing.

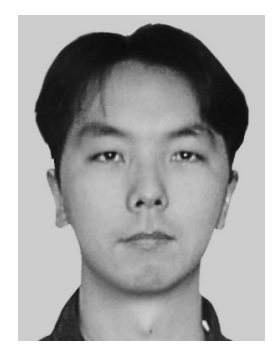

Go Hasegawa received his M.E. and Ph.D. degrees from Osaka University, Japan, in 1997 and 2000, respectively. From 1997 to 2000, he was a Research Assistant at the Graduate School of Economics, Osaka University. He is currently an Associate Professor at the Cybermedia Center, Osaka University. His research is in the area of transport architecture for future high-speed networks. He is a member of IEEE.

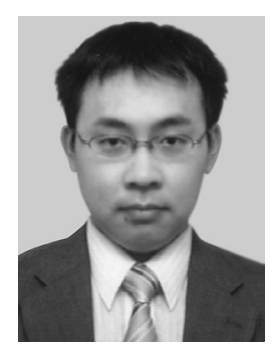

Yoshiaki Taniguchi received his B.E., M.E. and Ph.D. degrees from Osaka University, Japan, in 2002, 2004 and 2008, respectively. Since 2008, he has been an Assistant Professor at the Cybermedia Center, Osaka University. His research interests include wireless sensor networks, wireless mesh networks, and object tracking systems. He is a member of IEEE and IPSJ.

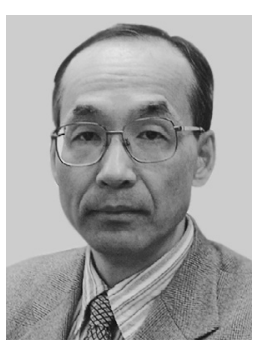

Hirotaka Nakano received his B.E., M.E. and D.E. degrees in Electrical Engineering from The University of Tokyo, Japan, in 1972, 1974 and 1977, respectively. He joined NTT Laboratories in 1977 and has been engaged in research and development of videotex systems and multimedia-on-demand systems. He was an executive manager of the Multimedia Systems Laboratory at the NTT Human Interface Laboratories from 1995 to 1999 . He was the head scientist of the Multimedia Laboratory at NTT DOCOMO until 2004 and is now a Professor at the Cybermedia Center, Osaka University. His research is in the area of ubiquitous networks. He is a member of IEEE and ITE. 